\title{
Intelligent Control Approaches for Modeling and Control of Complex Systems
}

\author{
José M. Andújar $\mathbb{D},{ }^{1}$ Eloy Irigoyen $\mathbb{D},{ }^{2}$ and Victor M. Becerra $\mathbb{D}^{3}$ \\ ${ }^{1}$ University of Huelva, Huelva, Spain \\ ${ }^{2}$ University of Basque Country, Bilbao, Spain \\ ${ }^{3}$ University of Portsmouth, Portsmouth, UK \\ Correspondence should be addressed to José M. Andújar; andujar@uhu.es
}

Received 10 May 2018; Accepted 10 May 2018; Published 31 May 2018

Copyright (C 2018 José M. Andújar et al. This is an open access article distributed under the Creative Commons Attribution License, which permits unrestricted use, distribution, and reproduction in any medium, provided the original work is properly cited.

A complex system can be defined as any system featuring a large number of interacting components whose aggregate activity is nonlinear and hence not derivable from the summations of the activity of individual components. As control methods are normally applied to dynamical systems, we are interested in complex dynamical systems. The rising complexity of man-made engineering systems (e.g., power networks, aircraft, autonomous vehicles, and communication networks) causes us increasing difficulties to understand their behaviour and to obtain accurate first-principle dynamical models to describe them, which reduces our capability to control those systems and make them behave in the way we want.

Intelligent control refers to approaches to control system design, modeling, identification, and operation that use artificial intelligence techniques, such as fuzzy logic, neural networks, machine learning, evolutionary computation, and genetic algorithms. Intelligent control techniques are often capable of controlling dynamical systems that, because of their complexity, are very difficult to control by other techniques. Intelligent control systems are characterized by attempts to emulate important aspects of biological intelligence. These aspects include, for instance, adaptation and learning, planning under uncertainty, and decision making. Intelligent control is interdisciplinary in nature as it seeks inspiration from biology, and it combines and extends theories and methods from areas such as control theory, mathematics, and computer science.

The aim of this special issue is to bring together a selection of the latest advances and trends in the application of intelligent control to the modeling and control of complex systems. This special issue consists of 12 contributions describing recent work from active researchers in the area.

$\mathrm{H}$. Ce et al. present a continuous finite-time robust control method for the trajectory tracking control of a nonholonomic wheeled mobile robot (NWMR). The approach involves an inner loop consisting of a conventional sliding mode controller and an outer loop that has a modified switched second-order sliding mode controller. Experiments are conducted on a Quanser QBot system. J. Zambrano et al. present an evolutionary approach for Wiener-Hammerstein system identification which can identify the linear and nonlinear sections of this nonlinear model structure in a single step, rather than two steps as is required in previous approaches. The proposed method is evaluated using wellknown benchmark data for nonlinear modeling.

J. M. Lozano Domínguez and T. D. Mateo Sanguino describe their work on the development of a fuzzy controller for an intelligent road signalling system consisting of a set of autonomous, intelligent, and low-cost devices that are employed to alert drivers when approaching zebra crossings if a pedestrian is traversing. The results of successful tests of the proposed signalling system on laboratory and real environments are described. C. González-Gutiérrez et al. present a neural network-based reconstructor for adaptive optics that is specially designed for multi-GPU systems.

V. San Juan et al. present a fuzzy logic-based approach for intelligent unmanned aerial vehicle (UAV) map generation and path planning for search and rescue operations. The approach is tested on a simulated search and rescue scenario 
based on real aerial images obtained during a forest fire in La Palma, Spain. A. Parra et al. provide an article on an intelligent torque vectoring approach for electric vehicles. The system consists of a neurofuzzy vertical tyre force estimator and a fuzzy yaw moment controller. A. Arroyo et al. present an approach for the development of neural models for imputation of missing ozone data in air quality datasets. Their approach is successfully tested using data from air quality stations based in Castilla y León, Spain.

T. D. Mateo Sanguino and J. C. Mendoza Betancourt describe an approach based on multiobjective genetic algorithms for the optimal modeling of wireless local area networks (WLANs). Their approach allows the investigation of various complex issues concerning the design of IEEE 802.11-based WLANs.

M. Marcano et al. present low speed longitudinal control algorithms for automated vehicles. They present a complete framework for automated driving, which includes the use of neurofuzzy techniques for longitudinal control and is implemented in virtual and real vehicles. Z. Zhou et al. describe an event-based time varying formation control for multiple UAVs that uses Markovian switching topologies. The effectiveness of the approach is assessed by means of simulations of six quadrotor UAVs flying in formation.

J. M. Gonzalez-Cava et al. present a fuzzy algorithm to introduce new variables in the drug supply decision-making process and test their proposed approach by using the analgesia nociception index (ANI) as a guiding variable for the analgesic process during surgical interventions. Finally, J. M. Torres and R. M. Aguilar describe an approach that uses deep learning to predict the future behaviour of complex systems and use power generation forecasting on a wind farm as a case study.

These authors highlight both the promise, the applications, the advantages brought about by the use of intelligent control approaches in modeling and control of complex systems, and challenges faced by engineers and scientists in developing and applying these techniques. In summary, this special issue provides a snapshot of very interesting and recent work on intelligent control approaches for modeling and control of complex systems.

José M. Andújar Eloy Irigoyen

Victor M. Becerra 


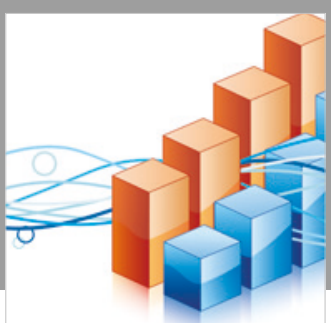

Advances in

Operations Research

\section{-n-m}
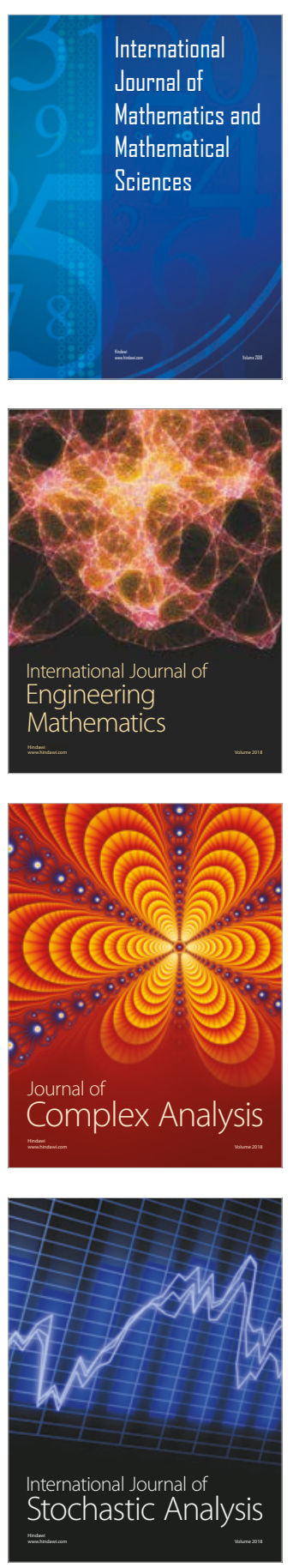
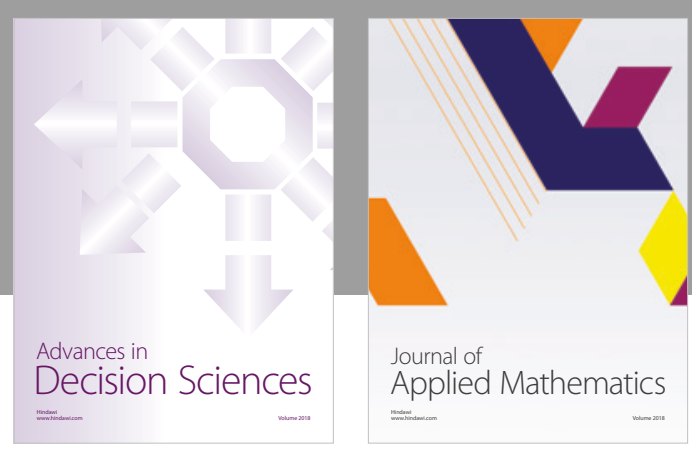

Journal of

Applied Mathematics
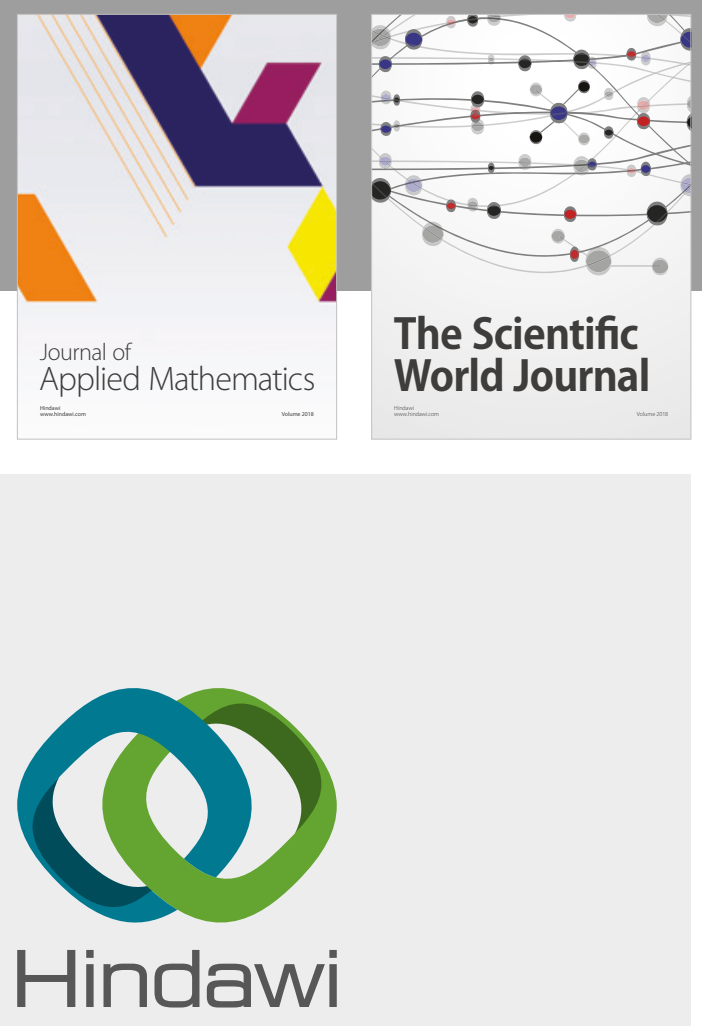

Submit your manuscripts at

www.hindawi.com

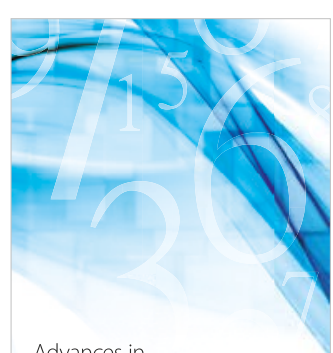

Advances in
Numerical Analysis
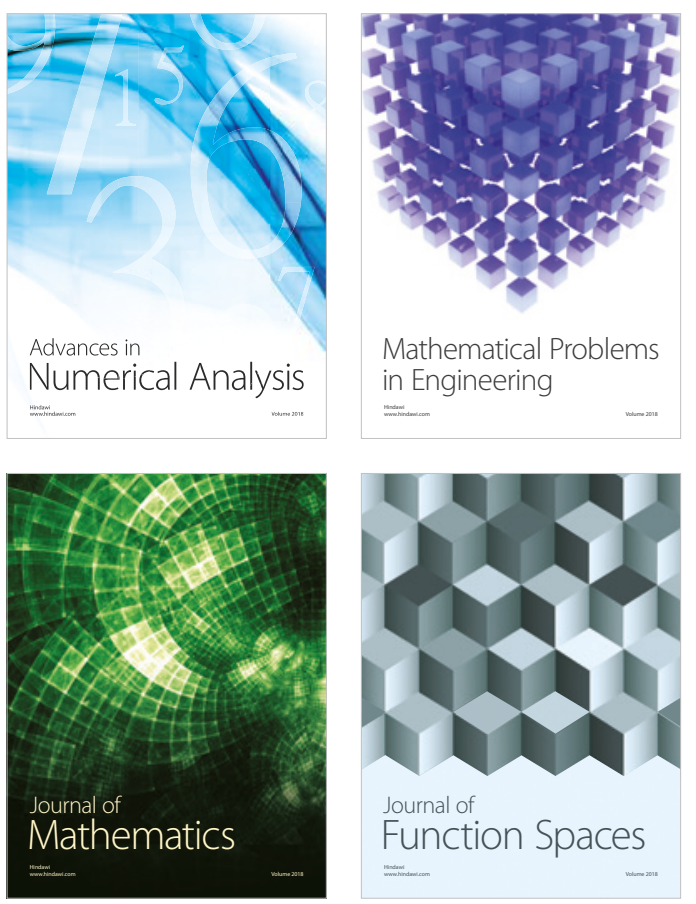

Mathematical Problems in Engineering

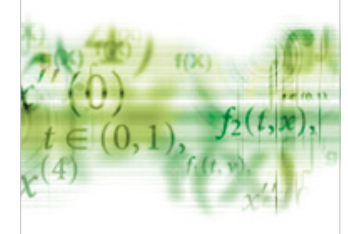

International Journal of

Differential Equations

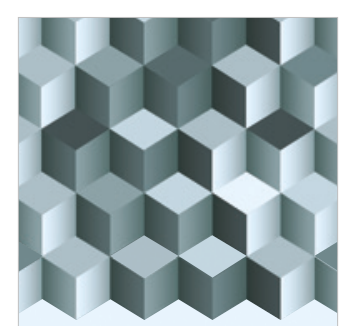

Journal of

Function Spaces
The Scientific

World Journal

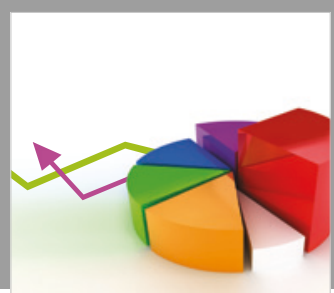

Journal of

Probability and Statistics
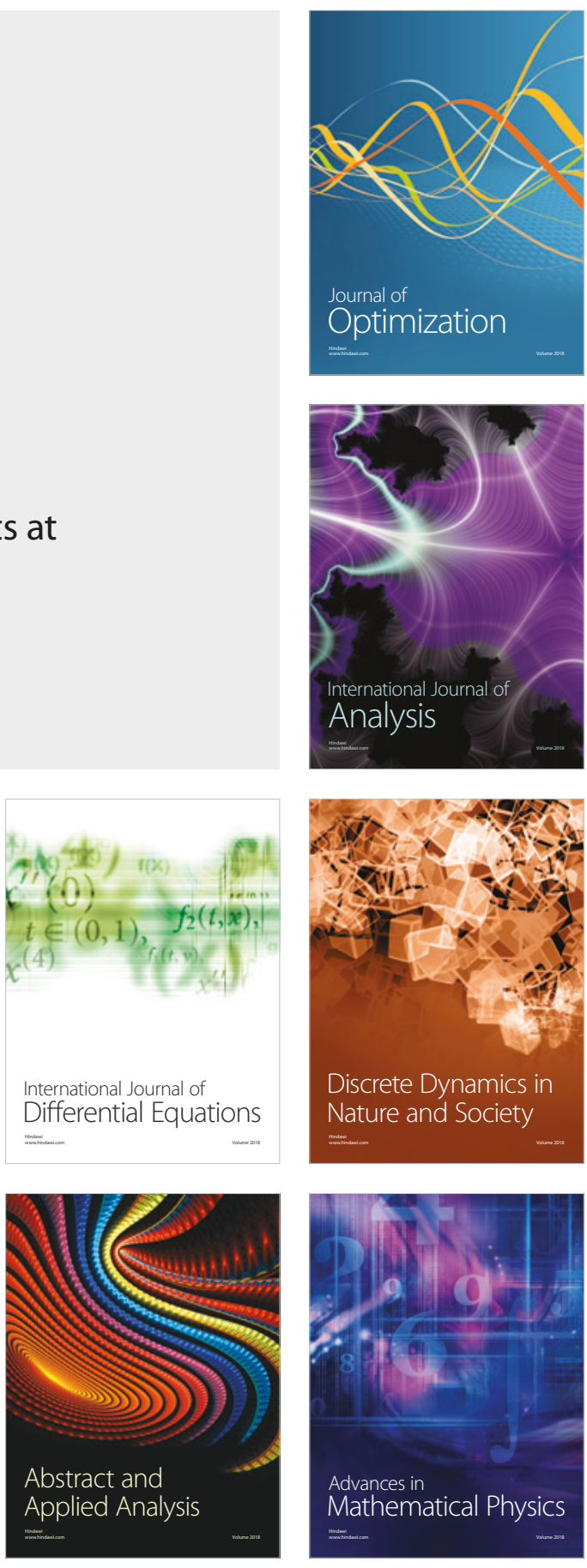